\title{
Metabolic Activity in Rat Tectal Grafts Is Influenced by Host Sensory Innervation
}

\author{
S. E. Dyson, A. R. Harvey, A. F. Stone, and G. A. Bell' \\ Department of Anatomy and Human Biology, University of Western Australia, Nedlands, W.A. 6009, and ${ }^{1}$ CSIRO Division \\ of Food Research, North Ryde, N.S.W. 2113, Australia
}

It has been shown previously that fetal tectal tissue grafted to the midbrain of newborn host rats grows, differentiates, and receives input from the host brain. In the present study, 4 neuroanatomical techniques have been combined to examine how metabolic activity in tectal transplants is influenced by an identified host sensory pathway.

Tectal tissue from E15 pigmented rat embryos was transplanted to the midbrain region of anesthetized newborn rats of the same strain. Six to 22 weeks later, the functional relationship between tectal transplants and the visual system of the host animal was examined by mapping metabolic activity in the grafts and relating this activity to the presence or absence of host retinal innervation. Metabolic activity in tectal grafts was assessed using the radioactive 2-deoxyglucose (2-DG) method and cytochrome oxidase (CO) histochemistry. Graft regions receiving input from host retinal axons were demonstrated by anterograde labeling after bilateral intraocular injections of HRP or WGA-HRP; all areas in grafts that were homologous to the superficial layers of normal superior colliculus (SC) were identified using AChE histochemistry.

The levels of metabolic activity demonstrated with 2-DG and $C O$ varied between animals and within individual grafts. Grafts that did not connect with the host showed only low metabolic activity. In grafts that received host input, localized areas of high metabolic activity were seen with both 2-DG and CO. Highest levels of activity were consistently found in areas containing both intense AChE activity and a high density of host retinal innervation. The enhanced metabolic activity in these retinorecipient areas of the graft was frequently similar to that seen in the stratum griseum superficiale (SGS) of host SC.

The increased metabolic activity seen in the retinorecipient areas of grafts may in part be attributable to presynaptic activity in the host optic terminals. However, there is evidence that high levels of $\mathrm{CO}$ activity are found in postsynaptic processes at retinal synapses and that 2-DG uptake is frequently associated with postsynaptic neural activity. It is argued, therefore, that host visual pathways that grow into grafts influence the metabolic and functional activity of retinorecipient cells in the grafted tissue.

\footnotetext{
Received May.27, 1987; accepted Oct. 8, 1987.

We are grateful to Delys Worthington and Alana Buck for their assistance with the preparation of figures for this paper. This work was supported by funding from the National Health and Medical Research Council of Australia and the CSIRO/ University of WA Collaborative Research Fund.

Correspondence should be addressed to Dr. S. E. Dyson at the above address. Copyright (C) 1988 Society for Neuroscience $0270-6474 / 88 / 051822-08 \$ 02.00 / 0$
}

Fetal neural tissue from many different areas of the developing mammalian brain has been successfully transplanted to the CNS of neonatal (e.g., Das, 1974; Lund and Hauschka, 1976; McLoon and Lund, 1980; Jaeger and Lund, 1981; Sunde and Zimmer, 1983; Harvey et al., 1984; Reier et al., 1986) or adult (e.g., Stenevi et al., 1976; Björklund and Stenevi, 1977; Kromer et al., 1981; Hallas, 1982; Oblinger and Das, 1982; McLoon and Lund, 1983; Sunde and Zimmer, 1983) hosts. The grafts survive, grow and differentiate within the host, and often form functional connections with the host brain. Electrophysiological studies have shown that grafts can alter host brain activity (Segal et al., 1981, 1985; Simons and Lund, 1985) and in many instances influcnce the behavior of the recipient animal (e.g., Perlow et al., 1979; Dunnett et al., 1982; Gash et al., 1982; Gage et al., 1983; Freed et al., 1984; Gibson et al., 1984; Buchanan and Nornes, 1986). The potential use of grafts in replacing damaged or deficient elements in the CNS has been widely documented.

For optimal integration of neural tissue grafts with the host brain, the grafts must themselves be functionally innervated by host nerve pathways. It has been shown that metabolic activity in substantia nigra grafts that reinnervate the neostriatum is equivalent to that in intact substantia nigra (Schmidt et al., 1982). Local glucose use in previously denervated hippocampus can also be restored by implantation of embryonic septal grafts, which provide new cholinergic innervation (Kelly et al., 1985). To date however, the influence of host innervation on neuronal activity in grafts has received little attention. This is a significant oversight, as the successful use of grafts for reconstruction of damaged brain pathways may be dependent on the establishment of effective reciprocal connections between the transplant and the host.

It has previously been shown that fetal tectal tissue transplanted to the midbrain region of neonatal rats receives input from many areas in the host brain (Harvey and Lund, 1981). At least 2 of these projections, from host retina (Lund and Hauschka, 1976; Lund and Harvey, 1981) and visual cortex (Harvey et al., 1982), form synapses on grafted tectal neurons. Electrophysiological studies have demonstrated that the host cortical input elicits excitatory responses from neurons in transplants; however, the functional efficacy of the host retinal projection remains unclear (Harvey et al., 1982).

Another way of studying how host primary visual pathways influence tectal graft activity is to relate host innervation to graft metabolism. In normal animals, retinorecipient areas such as the dLGN and superficial layers of the superior colliculus (SC) respond to alterations of visual sensory input with changed levels of metabolic activity. These changes can be demonstrated using the cytochrome oxidase (CO) (Wong-Riley, 1979; Wong-Riley 
and Riley, 1983) and radioactive 2-deoxyglucose (2-DG) (Miyaoka et al., 1979; McCulloch et al., 1980) techniques.

$\mathrm{CO}$ is a mitochondrial enzyme involved in the oxidative phosphorylation that yields ATP. In rat SC, $\mathrm{CO}$ activity is highest in the superficial gray and intermediate layers (Sandell, 1984). Uptake of 2-DG, a glucose analog, reflects metabolic activity in terms of glucose consumption and is coupled to the physiological activity of nerve pathways (Sokoloff et al., 1977). In the intact mammal the stratum griseum superficiale (SGS) of the SC, which receives visual input, always shows the highest metabolic activity. The deeper layers of the SC, which receive input from other sources, including the auditory and somatosensory systems, exhibit lower levels of activity.

In the present study, we have used the 2-DG and CO techniques to examine the degree of metabolic activity in tectal transplants. The relationship between graft metabolism and host retinal innervation has been directly assessed by injecting HRP or wheat-germ agglutinin-conjugated HRP (WGA-HRP) into the host eyes and comparing the distribution of labeled host retinal axons with the pattern of metabolic activity in the graft neuropil. It has been shown previously that AChE histochemistry can be used to identify areas in grafts homologous to the superficial layers of normal SC (Harvey and MacDonald, 1985). We have therefore employed this additional method to locate AChE-rich areas in the grafts and to determine how the metabolic activity in these specific regions is affected by the presence or absence of host retinal afferents. A preliminary account of this work has been published in abstract form (Dyson et al., 1987).

\section{Materials and Methods}

Rat fetuses of $15 \mathrm{~d}$ gestation (E15; day after mating, E0) were removed from anesthetized mothers of the hooded strain. Tectal tissue containing developing SC was removed from each fetus, stored in cool Ham's F10 culture medium, and then transplanted onto the midbrain of etheranesthetized newborn rats of the same strain (see Lund and Harvey, 1981 , for details).

Metabolic activity in the brains of 27 host animals was assessed 622 weeks after transplantation. Twenty-one animals were tested for 2-DG uptake; 5 of these animals were also examined for CO activity. The brains of 6 hosts were examined solely for $\mathrm{CO}$ activity.

2-DG experiments. The 21 host rats in this group were all anesthetized and received bilateral intraocular injections of HRP; 7 received 2.5-3.0 $\mu \mathrm{l}$ of $30 \%$ HRP (Boehringer Type I) in $2 \%$ dimethyl sulfoxide (DMSO) and 14 received $1.5-2.0 \mu \mathrm{l}$ of $1.5 \%$ WGA-HRP (Sigma) in distilled water. After $24 \mathrm{hr}$, each animal was injected intraperitoneally with 1.5 $\mathrm{mCi} / \mathrm{kg}$ of ${ }^{3} \mathrm{H}-2-\mathrm{DG}$ (Amersham). Animals were kept under 600 lux illumination (Miyaoka et al., 1979; McCulloch et al., 1980) for $45 \mathrm{~min}$, anesthetized, and then rapidly perfused. Several fixatives were used, all at room temperature in $0.1 \mathrm{M}$ phosphate buffer, $\mathrm{pH} 7.2-7.4$. Nine animals were perfused with $2.25-2.5 \%$ paraformaldehyde, and 12 with $1.5-2.0 \%$ paraformaldehyde $/ 1.0 \%$ glutaraldehyde. This fixative concentration was varied in an attempt to optimize the retention and localization of both 2-DG and HRP/WGA-HRP in the brain. After rapid perfusion, the brains were removed as quickly as possible and frozen in isopentane at $-70^{\circ} \mathrm{C}$. Some were sectioned immediately and others stored at $-90^{\circ} \mathrm{C}$ prior to tissue sectioning and processing. Grafts were not found in 3 hosts, and in another a graft was visible but clearly not attached to the host brain. These 4 brains were not processed further.

Seventeen brains containing transplants were sectioned on the cryostat at $-20^{\circ} \mathrm{C}$. A complete series of frozen sections ( $25 \mu \mathrm{m}$ thickness) of the transplant and underlying brain stem was prepared from each animal. The sections were collected in sets of 4 . The initial section of each set was thaw-mounted onto a glass slide, then rapidly dried and exposed to LKB Ultrofilm for 4-5 weeks. Autoradiographic tritium microscales (Amersham) were included with each film for calibration purposes. The remaining serial sections were stored in buffer at $4^{\circ} \mathrm{C}$. A section from each set of 4 was reacted for HRP uptake (tetramethyl- benzidine, TMB, method; Mesulam, 1978) and another for AChE activity (Karnovsky and Roots, 1964); the remaining section of the set was used either to repeat the HRP histochemistry, or (in 5 animals) to demonstrate $\mathrm{CO}$ activity (Wong-Riley, 1979).

Uptake of 2-DG into the superficial layers of SC in situ and its relation to retinal input was examined in one additional normal pigmented rat and in one rat bilaterally enucleated at birth. In these 2 animals, a $2.5 \%$ paraformaldehyde $/ 1.0 \%$ glutaraldehyde fixative was used. Tissue processing for 2-DG autoradiography was as described above.

Automated quantitative analysis of $2-D G$ autoradiographs was performed by digitizing the images using a videocamera and a DS65 board in an Apple II Plus microcomputer. Pixel data were thresholded across a nonlinear scale and printed out such that the range of optical densities in the autoradiograph was represented in the range of contours in the reconstructed image. The contour values can be converted to radiation data by reference to the optical densities of the autoradiographic standards. Pixel data analysis was facilitated by Super scan software (Micro Works). The computerized images shown in Figures 2 and 3 were produced by an enhancement routine that substitutes a pixel value by the mean of itself and the 8 adjacent pixel values, with the result that contoured regions are clarified (e.g., compare Fig. $1 D$ with $2 B$ ). In the filtered data, the edge can become fuzzy where a high-contrast region meets a low-contrast region; hence the area arrowed in Figure $2 B$ is closer to the edge of the tissue than is shown in this figure owing to the processing of the data.

Cytochrome oxidase ( $\mathrm{CO}$ ) experiments. In addition to the five 2-DG animals in which a series of tissue sections was also stained for $\mathrm{CO}$ histochemistry, 6 animals were perfused specifically for $\mathrm{CO}$ localization in tectal transplants. Under anesthesia, these rats received bilateral intraocular injections of $2.5-3.0 \mu \mathrm{l}$ of 30\% HRP in DMSO. After $24 \mathrm{hr}$, the animals were deeply anesthetized and were perfused with a solution of $2.5 \%$ paraformaldhyde, $1.5 \%$ glutaraldehyde, and $4.0 \%$ sucrose in $0.1 \mathrm{M}$ phosphate buffer, pH 7.2-7.4 (Wong-Riley, 1979). Brains were encapsulated in an albumen-gelatin mixture and $40 \mu \mathrm{m}$ frozen sections were cut on a sledge microtome. Parallel series of sections were processed for $\mathrm{CO}$, AChE, or HRP histochemistry, as described above.

\section{Results}

In this study we have combined 4 neuroanatomical techniques in order to examine directly how an identified host nerve pathway influences metabolic activity in CNS grafts. Simultaneous use of these techniques to visualize 2-DG uptake, anterograde WGA-HRP or HRP transport, CO activity, and AChE histochemistry was difficult and, to our knowledge, has not been attempted previously.

Grafts of tectal tissue that were implanted over the midbrain showed varied levels of metabolic activity. This variation occurred between animals and within individual grafts. Grafts that had no obvious connections with the host brain showed very low metabolic activity in comparison to the host's (e.g., Fig. $3 D$ ). In grafts that received input from the host, prominent foci of metabolic activity were seen with both CO and 2-DG. In 2-DG autoradiographs, these foci contrastcd sharply against the lower level of activity in the remainder of the graft. Localized areas containing the highest metabolic activity were studied closely in the semiadjacent serial sections stained for HRP and AChE.

\section{Relationship between retinal input and metabolic activity}

An example of high activity of the mitochondrial enzyme $\mathrm{CO}$ in a transplant situated over the host $\mathrm{SC}$ is shown in Figure $1 \mathrm{~A}$. A source of host neuronal input to this region was identified by the presence of HRP-labeled fibers derived from the host retina (Fig. 1B). This area, in turn, corresponded with a localized area of intense AChE staining (Fig. 1C). This series of micrographs (Fig. 1, $A-C$ ) demonstrates that when the region of the transplant that contains appropriate target tissue makes connections with 

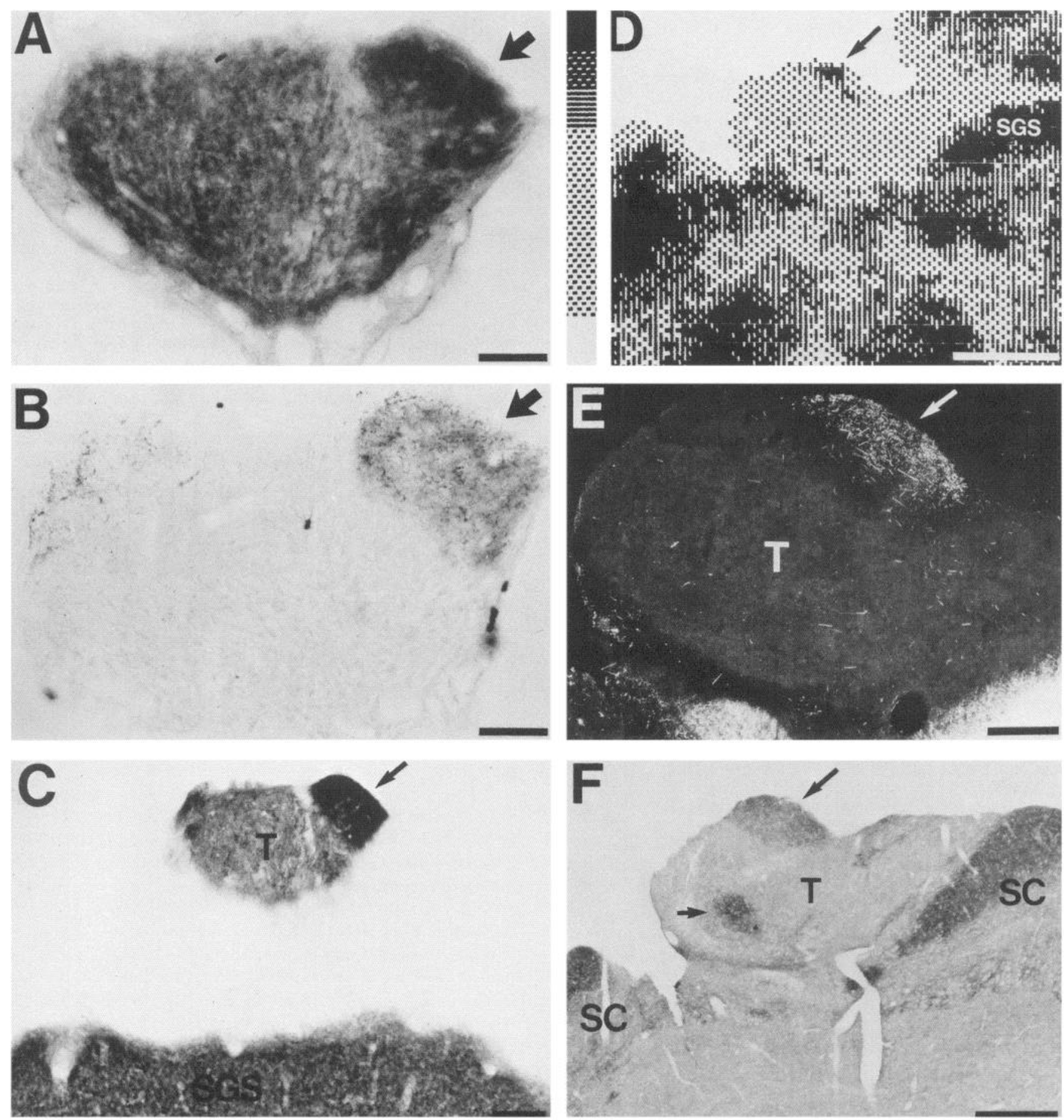

Figure 1. Photomicrographs demonstrating the relationship between retinal input and metabolic activity in 2 transplants. $A-C$, A transplant ( $T$ ) situated above the host SC. SGS, stratum griseum superficiale. Experiment M24. $A$, CO. Highest activity of this mitochondrial enzyme is seen in the right extremity of the transplant (arrow). B, TMB-HRP (light field). The location of HRP-labeled axons is arrowed. An area of less intense labeling is also seen on the left side of the graft. $C$, AChE. The localized area of intensely stained transplant tissue (arrow) is equivalent to the HRPlabeled region demonstrated in $B$ and the $C O$ patch seen in $A . D-F, A$ transplant $(T)$ attached in the midline at the level of the host superior colliculi $(S C)$. Experiment M33. Demonstration of metabolic activity using 2-DG mapping. D, 2-DG autoradiograph. Computerized activity contours. The area of the transplant showing high metabolic activity is arrowed. The optical-density calibration on the left represents metabolic activity in the section, with the highest activity at the top (black) and the lowest, equal to background noise, being white at the bottom of the scale. $E$, TMB-HRP (dark field). A localized area of heavily labeled retinal input on the upper surface is arrowed. $F$, AChE. A localized area of intensely stained tissue on the surface is arrowed; a more deeply placed dense "patch" is also present. The surface patch is equivalt nt to the retinorecipient area seen in $E$. Note, however, that the deeper patch does not receive any retinal input. Calibration bars: $50 \mu \mathrm{m}(A, B) ; 100 \mu \mathrm{m}(C) ; 1 \mathrm{~mm}(D)$; $200 \mu \mathrm{m}(E) ; 500 \mu \mathrm{m}(F)$. 

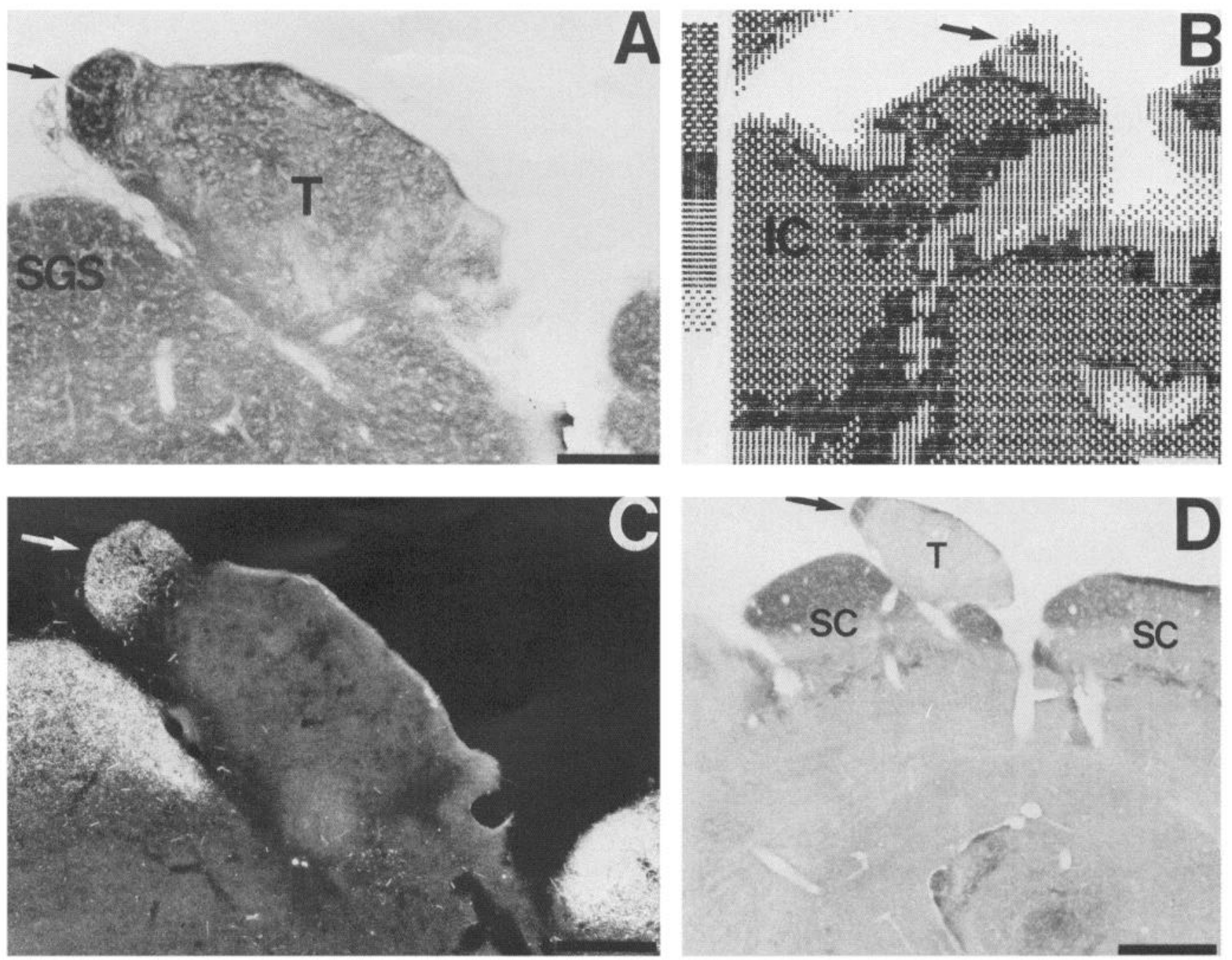

Figure 2. Correspondence between host input and functional activity in the graft tissue is demonstrated in a transplant attached over the left superior colliculus (SC). Experiment M34. A, CO. The region showing elevated activity of this mitochondrial enzyme is arrowed. SGS, stratum griseum superficiale. $B, 2-D G$ autoradiograph. Computerized activity contours. The area of the transplant showing high metabolic activity relative to surrounding tissue is arrowed. Highest activity is represented by the hatched texture at the top of the calibration scale. The lowest value (background) is white. IC, host inferior colliculus. $C$, TMB-HRP. Dark-field photomicrograph indicating the area receiving retinal input (arrow). $D, \mathrm{AChE}$. The corresponding localized area of intense staining for AChE is arrowed. Embedded transplant tissue is also located within the cerebral aqueduct. Calibration bars: $200 \mu \mathrm{m}(A, C) ; 500 \mu \mathrm{m}(B, D)$.

the host sensory system, high levels of CO enzymic activity can occur.

This correlation between the presence of appropriate target tissue, establishment of retinal connections, and high metabolic activity was also demonstrable with the metabolic marker 2-DG (Fig. 1, $D-F$ ). The computer enhancement of a 2-DG autoradiograph shows a localized area of high metabolic activity on the surface of a transplant (Fig. 1D). The level of activity in this region was equivalent to that seen in the adjacent host superficial SC layers. Comparison with a TMB-HRP-labeled section (Fig. $1 E$ ) and an AChE-stained section (Fig. $1 F$ ) revealed that the area in the graft containing high metabolic activity received host retinal input and contained intense $\mathrm{AChE}$ activity. A more deeply placed AChE-rich area was also evident (Fig. $1 F$ ). Note, however, that this deeper patch did not receive retinal input, and metabolic activity-although slightly higher than in the surrounding neuropil-was not localized in the same manner as was seen in the surface patch. Areas in grafts with the highest
2-DG uptake were always innervated by host retinal fibers. In 5 animals, processing for $\mathrm{CO}, \mathrm{AChE}, \mathrm{HRP}$, and 2-DG autoradiography was carried out. An example is shown in Figure 2. There was a clear correlation between high metabolic activity (shown by both the 2-DG and CO markers), AChE activity and host retinal innervation in this particular tectal graft.

It was necessary to establish that elevated metabolic activity (1) was not an intrinsic property of AChE-positive cells and (2) was dependent on the presence of specific connections with the host CNS. These points are demonstrated in Figure 3. A localized region of intense $\mathrm{AChE}$ activity located close to the aqueduct in an embedded transplant (Fig. $3 A$ ) did not receive retinal input. This was established with TMB-HRP tracing. CO activity in this easily definable region was extremely low (Fig. $3 B$ ). It is therefore clear that AChE-positive cells, which are presumably homologous to those seen in the SGS, did not necessarily have intrinsically high metabolic activity. The transplant depicted in Figure 3, $C, D$, lay over the host SC but had 

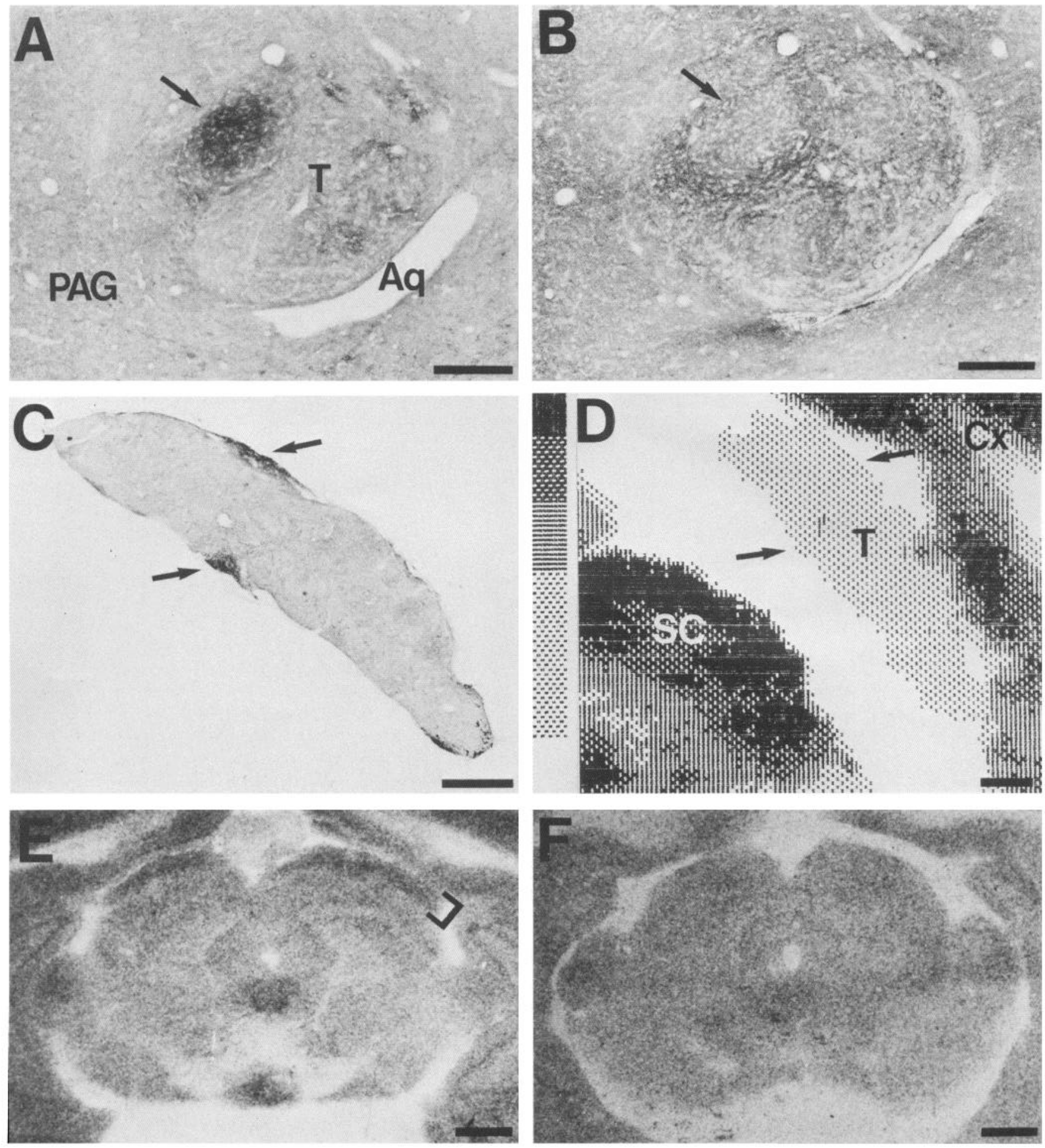

Figure 3. Demonstration that elevated metabolic activity (1) is not an intrinsic property of AChE-positive cells $(A, B$ and $C, D)$ and (2) is dependent on retinal input $(E, F) . A, B$, A deeply embedded transplant $(T)$ lying within the cerebral aqueduct $(A q)$. $P A G$, periaqueductal gray. $A$, AChE. A localized region of intense AChE activity is arrowed. TMB-HRP tracing established that this tissue did not receive retinal input. $B$, CO. The arrow indicates the position equivalent to that seen in $A$. Activity is quite low in this region; it is therefore clear that AChE-positive cells do not necessarily have intrinsically high metabolic activity. $C, D$, A transplant $(T)$ lying above the surface of the superior colliculus $(S C)$ ventromedial to the cerebral cortex $(C x)$. This graft received no host innervation. $C$, AChE. Localized patches of intense AChE activity (arrows) indicating potential retinorecipient cells. $D, 2$-DG autoradiograph. Computerized activity contours. It is clear that metabolic activity in the presumptive SGS cells demonstrated in $C$ does not rise above the low activity levels of the rest of the transplant when input with the host is not established. Highest activity at the top of calibration scale (black), lowest (white) at the bottom. $E, F, 2$-DG autoradiographs of midbrain sections from a control rat $(E)$ and from a rat enucleated bilaterally at birth $(F)$. E. Note the elevated metabolic activity in the superficial layers of the SC (square bracket). $F$, There is no corresponding intensification of silver grains in the SC. Calibration bars: $200 \mu \mathrm{m}(A, B) ; 500 \mu \mathrm{m}(C, D) ; 1 \mathrm{~mm}(E, F)$. 
no evident connections with the host CNS. TMB-HRP tracing was used to confirm the lack of connectivity with the retina. It can be seen from the computer enhancement of the 2-DG autoradiograph that, when input from the host was not established, metabolic activity in the presumptive SGS cells labeled with AChE did not rise above the low levels generally found throughout the graft neuropil.

A comparison of 2-DG autoradiographs showing midbrain sections obtained from a control rat (Fig. $3 E$ ) and from a rat enucleated bilaterally at birth (Fig. $3 F$ ) shows that elevated metabolic activity in the superficial layers of the normal superior colliculus is dependent on the presence of retinal input. There is nu definitive intensification of silver grains in the $\mathrm{SC}$ of the enucleated rat (Fig. $3 F$ ). Clearly, when retinal input is removed, metabolic activity in these layers is reduced in comparison to normal values. The correspondence between foci of elevated metabolic activity in the graft and the presence of host retinal input demonstrates that metabolism in the graft and sensory input from the host are closely interlinked in a manner analogous to that of the host tissue.

In one animal, a focal patch of raised 2-DG uptake was seen within a transplant that was embedded deep within the host midbrain, adjacent to the cerebral aqueduct. This graft did not receive any host retinal input. However, given that many regions in the host brain send axons into tectal tissue grafted into newborn hosts (Harvey and Lund, 1981), it is possible that this variation in metabolic activity was related to the presence of functional afferents from other, unidentified regions of the host brain (cf. Harvey et al., 1982).

\section{Discussion}

This study was undertaken to ascertain the possible influences of host input on metabolic activity within a tectal graft. A striking finding was the correlation of the sites of high levels of metabolic activity with retinorecipient foci in the graft.

In mammalian primary visual pathways it has been shown that there is normally a close correlation between metabolic and functional activity. For example, $\mathrm{CO}$ activity in macaque striate cortex decreases when physiological activity in retinofugal pathways is blocked with tetrodotoxin (Wong-Riley and Carroll, 1984). It has also been shown that, in normal rat SC, light stimulation (Miyaoka et al., 1979) or application of pharmacological agents such as apomorphine (McCulloch et al., 1980) increases 2-DG uptake in the superficial retinorecipient layers. In the absence of retinal input there is a substantial reduction of 2-DG uptake in the superficialSC (e.g., Fig. 3, E, F) (McCulloch et al., 1980).

In the present study, $\mathrm{CO}$ histochemistry and 2-DG autoradiography were used to provide information about the level of metabolic activity in tectal transplants. The levels of activity demonstrated with these techniques varied from one graft to another and, within a given transplant, there were local variations in 2-DG uptake and $\mathrm{CO}$ activity. Importantly, areas that showed the most marked increases in metabolic activity were correlated with patches that had high AChE activity and were innervated by host retinal axons. Indeed, in some instances, the levels of $\mathrm{CO}$ activity and 2-DG uptake found in these patches were qualitatively similar to those seen in the host superficial SC layers. The computerized activity contours of the 2-DG autoradiographs confirmed that this was the case, at least for glucose uptake-related metabolic activity. This is a significant observation, since it has previously been demonstrated that the density of optic synapses in tectal transplants can be as high as that found in the SGS of normal SC (Lund and Harvey, 1981). Note that cells in the localized AChE-dense areas in grafts that are believed to be homologous to the superficial retinorecipient layers of normal SC (Harvey and MacDonald, 1985) did not nccessarily have a high level of metabolic activity. Thus, high metabolic activity is not an intrinsic property of AChE-positive regions.

With regard to the specific influence of host retinal innervation on graft metabolism, it is clearly important to determine to what extent the increased metabolic activity in grafts reflects presynaptic or postsynaptic activity.

\section{Presynaptic versus postsynaptic location}

Electron-microscopic studies in other parts of the visual system suggest that most $\mathrm{CO}$ activity is located in mitochondria of the postsynaptic dendrites. In the LGN of the cat, $\mathrm{CO}$ activity in the neuropil increases as synapses on dendrites mature (Kageyama and Wong-Riley, 1986). This increased activity is principally due to increasing numbers of reactive mitochondria in the growing dendrites. In macaque lateral geniculate and striate cortex, much of the $\mathrm{CO}$ activity is also located in darkly reactive mitochondria in the dendrites (Hiltgen and Wong-Riley, 1986; Liu and Wong-Riley, 1986). Thus, in 2 regions of the visual system, it has been demonstrated that $\mathrm{CO}$ activity is dependent on enzyme activity in the postsynaptic region. In the case of the LGN, the input was from retinal axons, analogous to the situation with the grafts. It therefore appears reasonable to conclude that the elevated $\mathrm{CO}$ activity in areas of the graft that received retinal input was partly attributable to increased activity in the dendrites of the grafted cells. This conclusion is reinforced by the similarity between levels of enzyme activity in these areas and in the host SC.

It is not possible to directly ascertain the principal synaptic location of 2-DG activity. The tracer metabolite 2-DG-6-phosphate is soluble in water, and while it has been possible to record isotopic activity in electron-microscopic preparations (Buchner and Buchner, 1982; Benson et al., 1985), the resolution of these procedures does not allow reliable conclusions to be drawn about the synaptic location of metabolic activity. There is little doubt that presynaptic terminal activity contributes to observed 2-DG uptake. However, activity in the postsynaptic terminal is also considered to be important. In fact, physiological studies suggest that there is a tight coupling between 2-DG labeling and output spike activity (Schoppmann and Stryker, 1981; Theurich et al., 1984; cf. Lowel et al., 1987). The critical point of the present study is that levels of metabolic activity in retinorecipient areas of the transplant were, on occasion, very similar to those seen in the intact SGS. Thus, irrespective of the proportional contribution to total activity made by pre- and postsynaptic terminals, there was equivalence of metabolic activity in the host and transplant. This suggests that the retinally innervated areas of the transplant may be functioning in a manner analogous to that of host tissue.

In summary, this series of experiments demonstrates that the activity of cells in tectal grafts is modified by input from the host eyes. That is, input directed through a host sensory system is able to influence metabolic activity in areas of a transplant that have made appropriate connections. It is argued that the host afferents influence functional activity in postsynaptic cells in thcsc regions. In other CNS systems, grafts have been shown to affect neural activity in the host brain and to ameliorate 
physiological and behavioral deficits. In the longer term, it is important to determine whether neural transplants can establish a network of effective reciprocal connections leading to significant integration of the graft with the host brain.

\section{References}

Benson, T. E., G. D. Burd, C. A. Greer, D. M. D. Landis, and G. M. Shepherd (1985) High-resolution 2-deoxyglucose autoradiography in quick-frozen slabs of neonatal rat olfactory bulb. Brain Res. 339: 67-78.

Björklund, A., and U. Stenevi (1977) Reformation of the severed septohippocannpal pathway in the adult rat by transplanted septal neurons. Cell Tissue Res. 185: 289-302.

Buchanan, J. T., and H. O. Nornes (1986) Transplants of embryonic brainstem containing the locus coeruleus into spinal cord enhance the hindlimb flexion reflex in adult rats. Brain Res. 381: 225-236.

Buchner, S., and E. Buchner (1982) Functional neuroanatomical mapping in insects by [3H]2-deoxy- $d$-glucose at electron microscopical resolution. Neurosci. Lett. 28: 235-240.

Das, G. D. (1974) Transplantation of embryonic neural tissue in the mammalian brain. I. Growth and differentiation of neuroblasts from various regions of the embryonic brain in the cerebellum of neonate rats. TIT J. Life Sci. 4: 93-124.

Dunnett, S. B., W. C. Low, S. D. Iversen, U. Stenevi, and A. Björklund (1982) Septal transplants restore maze learning in rats with fornixfimbria lesions. Brain Res. 251: 335-348.

Dyson, S. E., A. R. Harvey, A. F. Stone, and G. A. Bell (1987) Mapping of metabolic activity in rat tectal grafts. Neurosci. Lett. 27: S71.

Freed, W. J., B. J. Hoffer, L. Olson, and R. J. Wyatt (1984) Transplantation of catecholamine-containing tissues to restore the functional capacity of the damaged nigrostriatal system. In Neural Transplants: Development and Function, J. R. Sladek, Jr., and D. M. Gash, eds., pp. 373-406, Plenum, New York.

Gage, F. H., S. B. Dunnett, U. Stenevi, and A. Björklund (1983) Aged rats: Recovery of motor impairments by intrastriatal nigral grafts. Science 221: 966-969.

Gash, D. M., P. H. Warren, L. E. Dick, J. R. Sladek, Jr., and J. R. Ison (1982) Behavioural modification in Brattleboro rats due to vasopressin administration and neural transplantation. Ann. NY Acad. Sci. 394: 672-681.

Gibson, M. J., D. T. Krieger, H. M. Charlton, E. A. Zimmerman, A.J. Silverman, and M. J. Perlow (1984) Mating and pregnancy can occur in genetically hypogonadal mice with preoptic area brain grafts. Science 225: 949-951.

Hallas, B. H. (1982) Transplantation into the mammalian adult spinal cord. Experientia 38: 699-701.

Harvey, A. R., and R. D. Lund (1981) Transplantation of tectal tissue in rats. II. Distribution of host neurons which project to transplants. J. Comp. Neurol. 202: 505-520.

Harvey, A. R., and A. M. MacDonald (1985) The development of acetylcholinesterase activity in normal and transplanted superior colliculus in rats. J. Comp. Neurol. 240: 117-127.

Harvey, A. R., G. T. Golden, and R. D. Lund (1982) Transplantation of tectal tissue in rats. III. Functional innervation of transplants by host afferents. Exp. Brain Res. 47: 437-445.

Harvey, A. R., J. B. Minson, M. J. Morris, and J. P. Chalmers (1984) Embryonic hypothalamic tissue transplanted to the IVth ventricle of newborn Brattleboro rats. Neurosci. Lett. 52: 269-274.

Hiltgen, G., and M. Wong-Riley (1986) Quantitative EM analysis of the effect of retinal impulse blockage on cytochrome oxidase activity in lamina IV of macaque striate cortex. Soc. Neurosci. Abstr. 12:130.

Jaeger, C. B., and R. D. Lund (1981) Transplantation of embryonic occipital cortex to the brain of newborn rats: A Golgi study of mature and developing transplants. J. Comp. Neurol. 200: 213-230.

Kageyama, G. H., and M. Wong-Riley (1986) The localization of cytochrome oxidase in the LGN and striate cortex of postnatal kittens. J. Comp. Neurol. 243: 182-194.

Karnovsky, M. J., and L. Roots (1964) A "direct-colouring" thiocholine method for cholinesterases. J. Histochem. Cytochem. 12: 219221.

Kelly, P. A. T., F. H. Gage, M. Ingvar, O. Lindvall, U. Stenevi, and A. Björklund (1985) Functional reactivation of the deafferented hip- pocampus by embryonic septal grafts as assessed by measurement of local glucose utilization. Exp. Brain Res. 58: 570-579.

Kromer, L. F., A. Björklund, and U. Stenevi (1981) Innervation of embryonic hippocampal implants by regenerating axons of cholinergic septal neurons in the adult rat. Brain Res. 210: 153-171.

Liu, S. Y., and M. Wong-Riley (1986) An electron microscopic analysis of the localization of cytochrome oxidase in the lateral geniculate nucleus of macaque monkeys. Soc. Neurosci. Abstr. 12: 1039.

Lowel, S., B. Freeman, and W. Singer (1987) Topographic organization of the orientation column system in large flat-mounts of the cat visual cortex: A 2-deoxyglucose study. J. Comp. Neurol. 255: 401-415.

Lund, R. D., and A. R. Harvey (1981) Transplantation of tectal tissue in rats. I. Organization of transplants and pattern of distribution of host afferents within them. J. Comp. Neurol. 201: 191-209.

Lund, R. D., and S. D. Hauschka (1976) Transplanted neural tissue develops connections with host rat brain. Science 193: 582-584.

McCulloch, J., H. E. Savaki, M. C. McCulloch, and L. Sokoloff (1980) Retina-dependent activation by apomorphine of metabolic activity in the superficial layer of the superior colliculus. Science 207: 313315.

McLoon, S. C., and R. D. Lund (1980) Specific projections of retina transplanted to rat brain. Exp. Brain Res. 40: 273-282.

McLoon, S. C., and R. D. Lund (1983) Development of fetal retina, tectum and cortex transplanted to the superior colliculus of adult rats. J. Comp. Neurol. 217: 376-389.

Mesulam, M.-M. (1978) Tetramethyl benzidine for horseradish peroxidase histochemistry: A non-carcinogenic blue reaction-product with superior sensitivity for visualizing neural afferents and efferents. $J$. Histochem. Cytochem. 26: 106-117.

Miyaoka, M., M. Shinohara, M. Batipps, K. D. Pettigrew, C. Kennedy, and L. Sokoloff (1979) The relationship between the intensity of the stimulus and the metabolic response in the visual system of the rat. Acta Neurol. Scand. (Suppl. 72) 60:16-17.

Oblinger, M. M., and G. D. Das (1982) Connectivity of neural transplants in adult rats: Analysis of afferents and efferents of neocortical transplants in the cerebellar hemispheres. Brain Res. 249: 31-49.

Perlow, M. J., W. J. Freed, B. J. Hoffer, A. Seiger, L. Olson, and R. J. Wyatt (1979) Brain grafts reduce motor abnormalities produced by destruction of nigrostriatal dopamine system. Science 204: 643-647.

Reier, P. J., B. S. Bregman, and J. R. Wujek (1986) Intraspinal transplantation of embryonic spinal cord tissue in neonatal and adult rats. J. Comp. Neurol. 247: 275-296.

Sandell, J. H. (1984) The distribution of hexokinase compared to cytochrome oxidase and acetylcholinesterase in the somatosensory cortex and the superior colliculus of the rat. Brain Res. 290: 384 389.

Schmidt, R. H., M. Ingvar, O. Lindvall, U. Stenevi, and A. Björklund (1982) Functional activity of substantia nigra grafts reinnervating the striatum: Neurotransmitter metabolism and (14C) 2-deoxy-Dglucose autoradiography. J. Neurochem. 38: 737-748.

Schoppmann, A., and M. P. Stryker (1981) Physiological evidence that the 2-deoxyglucose method reveals orientation columns in cat visual cortex. Nature 293: 574-576.

Segal, M., U. Stenevi, and A. Björklund (1981) Reformation in adult rats of functional septo-hippocampal connections by septal neurons regenerating across an embryonic hippocampal tissue bridge. Neurosci. Lett. 27: 7-12.

Segal, M., A. Björklund, and F. H. Gage (1985) Transplanted septal neurons make viable cholinergic synapses with a host hippocampus. Brain Res. 336: 302-307.

Simons, D. J., and R. D. Lund (1985) Fetal retinae transplanted over tecta of neonatal rats respond to light and evoke patterned neuronal discharges in the host superior colliculus. Dev. Brain Res. 21: 156159.

Sokoloff, L., M. Reivich, C. Kennedy, M. H. DesRosiers, C. S. Patlak, K. Pettigrew, O. Sakurada, and M. Shinohara (1977) The ${ }^{14} \mathrm{C}$ deoxyglucose method for the measurement of local cerebral glucose utilization: Theory, procedure and normal values in the conscious and anesthetized albino rat. J. Neurochem. 28: 897-916.

Stenevi, U., A. Björklund, and N-A. Svendgaard (1976) Transplantation of central and peripheral monoamine neurons to the adult rat brain: Techniques and conditions for survival. Brain Res. 114: 1-20.

Sunde, N. A., and J. Zimmer (1983) Cellular, histochemical and connective organization of the hippocampus and fascia dentata transplanted to different regions of immature and adult rat brains. Dev. Brain Res. 8: 165-191. 
Theurich, M., C. M. Muller, and H. Scheich (1984) 2-Deoxyglucose accumulation parallels extracellularly recorded spike activity in the avian auditory neostriatum. Brain Res. 332: 157-161.

Wong-Riley, M. T. T. (1979) Changes in the visual system of monocularly sutured or enucleated cats demonstrable with cytochrome oxidase histochemistry. Brain Res. 171: 11-28.
Wong-Riley, M., and E. Carroll (1984) Effect of impulse blockage on cytochrome oxidase activity in monkey visual system. Nature 307 : 262-264.

Wong-Riley, M., and D. Riley (1983) The effect of impulse blockage on cytochrome oxidase activity in the cat visual system. Brain Res. 261: $185-193$. 